\title{
Vancomycin-resistant Enterococci in Intensive Care Hospital Settings
}

\author{
Daren J Austin/ ${ }^{+}$, Marc JM Bonten*
}

\begin{abstract}
Wellcome Trust Centre for the Epidemiology of Infectious Diseases, University of Oxford, South Parks Road, Oxford OX1 3PS, UK *Department of Internal Medicine, University Hospital Utrecht, PO Box 85000, 3508, GA Utrecht, Netherlands
\end{abstract}

Vancomycin-resistant enterococci (VRE) have recently emerged as a nosocomial pathogen and present an increasing threat to the treatment of severely ill patients in intensive-care hospital settings. We outline results of a study of the epidemiology of VRE transmission in ICUs and define a reproductive number $R_{0}$; the number of secondary colonization cases induced by a single VRE-colonized patient in a $V R E$-free ICU, for VRE transmission. For VRE to become endemic requires $R_{0}>1$. We estimate that in the absence of infection control measures $R_{0}$ lies in the range 3-4 in defined ICU settings. Once infection control measures are included $R_{0}=0.6$, suggesting that admission of VRE-colonized patients can stabilize endemic VRE.

Key words: vancomycin resistance - transmission dynamics - epidemiology - infection control

Increasing antibiotic resistance in common bacterial pathogens presents a growing threat worldwide. The emergence of vancomycin-resistant enterococci (VRE) as a nosocomial pathogen is a striking example of this new danger to vulnerable patients. In both the United States and the United Kingdon, the frequency with which isolates have been recovered has increased dramatically during the past seven years (CDC 1995, PHLS 1996). For patients infected with VRE treatment options are often limited and control of outbreaks relies heavily on conventional infection control procedures (Husani \& Raad 1997).

Molecular epidemiological studies of early endemic infections suggested that single clones were primarily responsible (Boyce et al. 1995). More recently, many outbreaks appear to involve more than one clone, indicating reintroduction (Morris et al. 1995, Slaughter et al. 1996, Bonten et al. 1996). Since enterococci form part of our natural flora, it was believed that new hospital outbreaks were a result of endogenous sources. However recent studies have revealed that transmission of VRE via the hands of transiently colonized heath-care workers (HCWs) is a very important determinant of spread and persistence (Bonten et al. 1996).

DJA thanks the Wellcome Trust for continued support. MJMB thanks Merck Sharpe \& Dohme and the Infectious Diseases Society of the Netherlands and Flanders for grant support.

${ }^{+}$Corresponding author. Fax: +44-1865-281245. E-mail: daren.austin@zoology.oxford.ac.uk

Received 15 June 1998

Accepted 30 July 1998
In a previous study conducted at the Cook County Hospital (CCH) Chicago, IL, that measured the relative efficacies of various barrier infection control precautions (Slaughter et al. 1996) rectal cultures were taken daily and environmental cultures monthly (Slaughter et al. 1996). Using pulsedfield gel electrophoresis, a total of 19 strain types of VRE were identified. In a follow-up study it was subsequently confirmed that patients rather than the environment provide the major reservoir of VRE (Bonten et al. 1996).

The transmission dynamics of VRE in an ICU setting can be represented by a set of coupled differential equations with framework summarized by Fig. If we view patients as definitive hosts and HCWs as vectors for transmission, then the structure of the model reduces to that of the RossMacdonald equations for malaria transmission (Anderson \& May 1991). A central concept in infectious disease transmission is the reproductive number $R_{0}$; the number of secondary cases of VRE colonization generated by a single primary case in a VRE-free ICU. If $R_{0}>1$ an outbreak of VRE will persist and become endemic with prevalence 1-1/ $R_{0}$. If $R_{0}<1$ the outbreak will fade to extinction. For indirect VRE transmission via HCWs $R_{0}$ is defined as

$$
R_{0}=m b_{p} b_{s} r^{2} D_{p} D_{s}
$$

where $m$ is the staff-patient ratio, $b_{p}$ and $b_{s}$ are the respective probabilities of transmission from HCW-patient and vice-versa, $r$ is the staff-patient contact rate (patient contacts per unit time) and parameters $D_{s}$ and $D_{p}$ represent the average duration VRE remains transmissible on the hands of HCWs (typically one hour) and from patients (typi- 
cally the duration of their stay in the ICU i.e., days). The contact rate appears as a squared quantity reflecting the patient-HCW-patient nature of transmission.

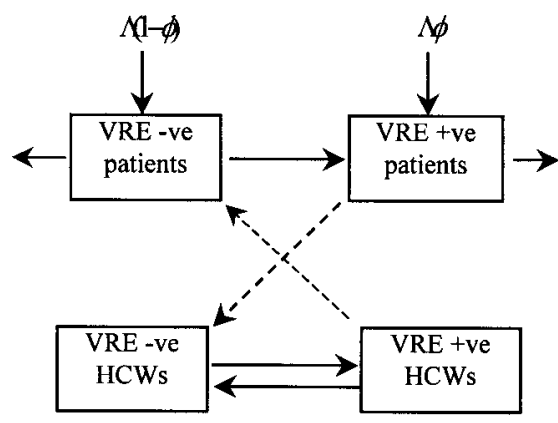

Model of indirect patient-health care workers (HCW)-patient vancomycin-resistant enterococci (VRE) transmission. Patients are admitted at a rate $L$ per day with a fraction $f$ already colonized. Dashed arrows indicate indirect transmission. Colonized patients remain in the ICU for duration $D_{p}$ and HCWs can transmit VRE for duration $D_{s}$.

Infection control measures will influence $R_{0}$ in different ways. Barrier precautions such as handwashing reduce the probability of HCWs transmitting VRE, once colonized, by a factor (1-p) where $p$ is the observed compliance with hand-washing measures. Cohorting HCWs will reduce the effective staff-patient ratio $m$ by a factor $(1-q)$ where $q$ is the proportion of staff cohorted to a single patient. Increasing the numbers of HCWs will increase $m$ but may reduce the patient contact rate $r$ and hence $R_{0}$.

Estimates of compliance with barrier precautions have been reported as less than 50\% (Doebbbelling et al. 1992), suggesting that VRE will not controlled if $R_{0}>2$. Cohorting of staff members via one-to-one nursing can give very high cohorting levels, perhaps $80 \%$ with a correspondingly higher eradication $R_{0}<5$. Indeed outbreaks have been brought under control using just such methods (see e.g., Haley et al. 1995).

During the course of a 133-day study of endemic VRE at CCH a mean compliance of $51 \%$ was observed and the level of cohorting of HCWs was estimated to be $80 \%$. The mean endemic prevalence of VRE was found to be $36 \%$ (95\% CI 3-68) and $15 \%$ of patients were already colonized on admission (Slaughter et al. 1996). Our analyses indicate that the effective reproductive number (including infection control measures) $R_{0}(p, q)=0.6$, corresponding to a true reproductive number $R_{0}=3.11$ in the absence of infection control measures. We deduce that since $R_{0}(p, q)<1$, infection control would ordinarily control VRE. However the admission of colonized patients continued to stabilize endemic VRE. The observed reduction in VRE transmission is considerable. In the absence of infection control, the predicted endemic prevalence of VRE is $75 \%$ compared with an observed mean of $36 \%$.

The use of molecular epidemiology has demonstrated that the primary determinant of endemic VRE is indirect patient-HCW-patient transmission, rather than environmental or endogenous sources. Using a precise mathematical framework enables careful analysis of the transmission dynamics of VRE and allows for quantitative measurements of both transmission and, more importantly, intervention can be made. As treatment options become more limited, clinicians will become ever more reliant on conventional infection control procedures. The quantitative measurements outlined can be used to assist in enabling better management of limited resources to combat the threat of VRE in ICU hospital settings.

\section{REFERENCES}

Anderson RM, May RA 1991. Infectious Diseases of Humans: Dynamics and Control, Oxford University Press, $755 \mathrm{pp}$.

Bonten MJM, Hayden MK, Nathan J, van Voorhis J, Matushek M, Slaughter S, Rice T, Weinstein RA 1996. Epidemiology of colonization of patients and environment with vancomycin-resistant enterococci. Lancet 348: 1615-1619.

Boyce JM, Mermal LA, Zervos MJ, Rice LB, PotterBynoe G, Giorgio C, Medeiros AA 1995. Controlling vancomycin-resistant enterococci. Infect Control Hosp Epidemiol 16: 634-637.

CDC 1995. Recommendations for preventing the spread of vancomycin-resistance. MMER 44 (RR12): 1-13.

Doebbelling BN, Stanley GL, Sheetz CT, Pfaller MA, Houston AK, Annis L, Li N, Wenzel RP 1992. Comparison of efficacy of alternative hand-washing agents in reducing nosocomial infections in intensive-care units. NEJM 327: 88-93.

Haley RW, Cushion NB, Tenover FC, Bannerman TL, Dryer D, Ross J, Sanchez PJ, Siegel JD 1995. Eradication of endemic methicillin-resistant Staphylococcu aureus from a neonatal intensive-care unit. J Infect Dis 171: 614-624.

Husani R, Raad I 1997. Treatment and prevention of vancomycin-resistant enterococcus. Curr Opin Intensive Care 10: 431-434.

Morris Jr JG, Shay DK, Hebden JN 1995. Enterococci resistant to multiple antimicrobial agents, including vancomycin: establishment of endemicity in a University Medical Center. Ann Internal Med 123: 250259.

PHLS 1996. Vancomycin-resistant enterococci in hospitals in the United Kingdom. CDR Weekly 6: 1.

Slaughter S, Hayden MK, Nathan C, Hu TC, Rice TJ, van-Voortis J, Matusheka M, Franklin C, Weinstein RA 1996. A comparison of the universal use of gloves and gowns with that of glove use alone on the acquisition of vancomycin-resistant enterococci in a medical intensive-care unit. Ann Internal Med 125: 448-456. 